\title{
Flexoelectric Instability in Nematic Liquid Crystal between Coaxial Cylinders
}

\author{
I. V. KOTOV ${ }^{a}$, M. V. KHAZIMULLIN ${ }^{a}$ and A. P. KREKHOV ${ }^{a, b}$ \\ ${ }^{a}$ Institute of Molecule and Crystal Physics, Russian Academy of \\ Sciences, 450025 Ufa, Russia; ${ }^{b}$ Physikalisches Institut, Universität \\ Bayreuth, D-95440 Bayreuth, Germany
}

The stability of the equilibrium configurations of a nematic liquid crystal confined between two coaxial cylinders is analysed when a radial electric field is applied and the flexoelectric effect is taken into account. The threshold for perturbations depending only on the radius $r$ in the cylindrical coordinate system and strong boundary conditions is studied. A new type of orientational transition caused by pure flexoelectric effect is found.

Keywords: nematic liquid crystal, flexoelectric instability

\section{INTRODUCTION}

The uniform orientation of nematic liquid crystal (NLC) is caused by the orienting action of appropriately treated confining plates, which define a fixed orientation of the NLC at the boundary. When an electric or magnetic field is applied to such NLC sample in an appropriate direction, a director reorientation via a Fréedericksz transition may result, which has been studied extensively in case of a plane layer geometry.

For a NLC confined between two coaxial cylinders the orientational transition may occur even in the absence of external fields depending on the ratio of the radii of the inner cylinder to that of the outer one [1, 2]. The Fréedericksz transition in this geometry was analysed for a magnetic field directed radially outwards from the common axis of the cylinders [3] and tangentially to circles in the plane perpendicular to the cylinders' axis [4, 5] as well as for a radial electric field. Here a voltage was applied between the two 
coaxial cylinders [6, []. In this geometry the critical voltage for the Fréedericksz transition depends on the ratio between the cylinders radii.

In this paper we analyse the influence of the flexoelectric effect on the orientational transitions in a NLC confined between two coaxial cylinders under applied d.c. radial electric field. The flexoelectricity describes the linear coupling between an applied electric field and gradients in the director field [8]. In contrast to the case of a plane nematic layer, in cylindrical geometry under applied electric field one has a contribution from the flexoelectric effect in the bulk torque acting on the director. Here we calculate the critical voltage for the orientational transition where the director distribution $\hat{\mathbf{n}}=\left(n_{r}, n_{\phi}, n_{z}\right)$ depends only on the radius $r$ in the cylindrical coordinate system $(r, \phi, z)$ with $z$ axis along the axis of cylinders. Strong boundary conditions are imposed. Two typical initial director distributions [homeotropic orientation $\hat{\mathbf{n}}=(1,0,0)$ and planar one $\hat{\mathbf{n}}=(0,1,0)$ ] are considered. It is found that for planar orientation a new type of orientational transition caused purely by the flexoelectric effect takes place.

\section{BASIC EQUATIONS}

Let us consider the nematic enclosed between two infinite coaxial cylindrical electrodes whose inner and outer radii are $r_{1}$ and $r_{2}$, respectively. The director distribution $\hat{\mathbf{n}}=\hat{\mathbf{n}}(r)$ depends only on $r$. The voltage applied between the electrodes provides the radially directed electric field $\mathbf{E}=(E, 0,0)$. The equilibrium director configuration can be found from the extremum condition of the total free energy (per unit length of cylinders)

$$
\mathcal{F}=2 \pi \int_{r_{1}}^{r_{2}}\left(F_{d}+F_{e l}+F_{f l}\right) r d r .
$$

Here $F_{d}$ is the elastic energy density

$$
F_{d}=\frac{1}{2}\left[K_{1}(\nabla \cdot \hat{\mathbf{n}})^{2}+K_{2}(\hat{\mathbf{n}} \cdot(\nabla \times \hat{\mathbf{n}}))^{2}+K_{3}(\hat{\mathbf{n}} \times(\nabla \times \hat{\mathbf{n}}))^{2}\right] .
$$

The electric field contribution is given by

$$
F_{e l}=-\frac{1}{2} \epsilon_{0} \epsilon_{a}(\hat{\mathbf{n}} \cdot \mathbf{E})^{2},
$$


where $\epsilon_{a}=\epsilon_{\|}-\epsilon_{\perp}$ is the dielectric anisotropy and the radial component of the electric field $E=U /\left[r \ln \left(r_{2} / r_{1}\right)\right]$ with $U=U\left(r_{1}\right)-U\left(r_{2}\right)$ the voltage drop across the cylinders. The contribution of flexoelectricity

$$
F_{f l}=-(\mathbf{E} \cdot \mathbf{P}), \quad \mathbf{P}=e_{11} \hat{\mathbf{n}}(\nabla \cdot \hat{\mathbf{n}})-e_{33}(\hat{\mathbf{n}} \times(\nabla \times \hat{\mathbf{n}}))
$$

is connected with flexo-polarization $\mathbf{P}$ induced by the splay and bend deformations of the director field and $e_{11}, e_{33}$ are the flexoelectric coefficients.

Minimisation of (1) with the normalisation $\hat{\mathbf{n}}^{2}=1$ gives the torque balance equation

$$
[\hat{\mathbf{n}} \times \mathbf{h}]=0, \quad h_{i}=\frac{d}{d r}\left(\frac{\partial f}{\partial n_{i, r}}\right)-\frac{\partial f}{\partial n_{i}}, \quad i=r, \phi, z
$$

where $f=\left(F_{d}+F_{e l}+F_{f l}\right) r$. One can easily verify that $\hat{\mathbf{n}}_{0}=(1,0,0)$ and $\hat{\mathbf{n}}_{0}=(0,1,0)$ are solutions of equation (5) for homeotropic and planar boundary conditions, respectively.

\section{STABILITY ANALYSIS}

Let us now examine the stability of these configurations.

I) Homeotropic anchoring with boundary conditions

$$
\hat{\mathbf{n}}\left(r=r_{1}\right)=\hat{\mathbf{n}}\left(r=r_{2}\right)=(1,0,0) .
$$

Small deviations from the exact solution $\hat{\mathbf{n}}_{0}=(1,0,0)$ of (5) can be written as

$$
\hat{\mathbf{n}}=\hat{\mathbf{n}}_{0}+\delta \hat{\mathbf{n}}=\left[1-\frac{1}{2}\left(\delta n_{\phi}^{2}+\delta n_{z}^{2}\right) ; \delta n_{\phi} ; \delta n_{z}\right]
$$

up to second order in the perturbations $\delta n_{\phi}, \delta n_{z}$ which depend only on $r$. Then for the change of total free energy one obtain up to second order in $\delta \hat{\mathbf{n}}$

$$
\begin{aligned}
& \Delta \mathcal{F}_{\text {hom }} \equiv \mathcal{F}\left(\hat{\mathbf{n}}_{0}\right)-\mathcal{F}\left(\hat{\mathbf{n}}_{0}+\delta \hat{\mathbf{n}}\right)= \\
&=2 \pi \int_{r_{1}}^{r_{2}}\left\{\frac{1}{2} K_{1}\left[-\frac{1}{r^{2}}\left(\delta n_{\phi}^{2}+\delta n_{z}^{2}\right)\right]+\frac{1}{2} K_{3}\left[\delta n_{\phi}^{\prime 2}+\delta n_{z}^{\prime 2}+\frac{1}{r^{2}} \delta n_{\phi}^{2}\right]+\right. \\
& \frac{1}{2} \epsilon_{0} \epsilon_{a} \tilde{U}^{2} \frac{1}{r^{2}}\left(\delta n_{\phi}^{2}+\delta n_{z}^{2}\right)+ \\
&\left.\tilde{U} e_{11} \frac{1}{r^{2}}\left(\delta n_{\phi}^{2}+\delta n_{z}^{2}\right)+\tilde{U} e_{33} \frac{1}{r^{2}} \delta n_{\phi}^{2}\right\} r d r
\end{aligned}
$$


where the boundary conditions $\delta \hat{\mathbf{n}}\left(r=r_{1}\right)=\delta \hat{\mathbf{n}}\left(r=r_{2}\right)=0$ were taken into account, $\tilde{U}=U / \ln \left(r_{2} / r_{1}\right)$ and $f^{\prime} \equiv d f / d r$.

The necessary and sufficient condition for $\Delta \mathcal{F}_{\text {hom }}$ to be positive definite is that the Euler-Lagrange equations

$$
\begin{aligned}
& K_{3} \frac{d^{2}}{d \alpha^{2}} \delta n_{\phi}+\left[K_{1}-K_{3}-\epsilon_{0} \epsilon_{a} \tilde{U}^{2}-2 \tilde{U}\left(e_{11}+e_{33}\right)\right] \delta n_{\phi}=0 \\
& K_{3} \frac{d^{2}}{d \alpha^{2}} \delta n_{z}+\left[K_{1}-\epsilon_{0} \epsilon_{a} \tilde{U}^{2}-2 \tilde{U} e_{11}\right] \delta n_{z}=0
\end{aligned}
$$

have nonzero solutions $\delta n_{\phi}, \delta n_{z} \neq 0$ in the range $0<\alpha<\ln \left(r_{2} / r_{1}\right)$ where we introduced the new variable $\alpha=\ln \left(r / r_{1}\right)$. Since equations (9), (10) are uncoupled one gets two independent conditions for the instability of initial homeotropic orientation with respect to $\delta n_{\phi}$ and $\delta n_{z}$ perturbations. Solving (9), (10) one obtains for the critical voltage $U_{c}$ for the two types of orientational transitions in cylindrical geometry

$$
\begin{gathered}
\delta n_{\phi}: \quad-\operatorname{sign}\left(\epsilon_{a}\right)\left(\frac{U_{c}}{U_{F_{3}}}\right)^{2}-\left(\frac{U_{c}}{U_{F_{3}}}\right) \frac{2\left(e_{11}+e_{33}\right) \ln \left(r_{2} / r_{1}\right)}{\pi \sqrt{K_{3} \epsilon_{0}\left|\epsilon_{a}\right|}}= \\
=1+\frac{K_{3}-K_{1}}{K_{3}}\left(\frac{\ln \left(r_{2} / r_{1}\right)}{\pi}\right)^{2}, \\
\delta n_{z}: \quad-\operatorname{sign}\left(\epsilon_{a}\right)\left(\frac{U_{c}}{U_{F_{3}}}\right)^{2}-\left(\frac{U_{c}}{U_{F_{3}}}\right) \frac{2 e_{11} \ln \left(r_{2} / r_{1}\right)}{\pi \sqrt{K_{3} \epsilon_{0}\left|\epsilon_{a}\right|}}= \\
=1-\frac{K_{1}}{K_{3}}\left(\frac{\ln \left(r_{2} / r_{1}\right)}{\pi}\right)^{2}
\end{gathered}
$$

where $U_{F_{3}}=\pi \sqrt{K_{3} / \epsilon_{0}\left|\epsilon_{a}\right|}$ is the threshold voltage for the bend Fréedericksz transition in case of a plane NLC layer. In Figure 11 the two types of transitions correspond to $\delta n_{\phi}$ and $\delta n_{z}$ perturbations are shown schematically. In the absence of electric field $(U=0)$ there is a possibility of orientational transition for a radius ratio above some critical value. The transition corresponding to $\delta n_{z}$ perturbation [Fig. 1(b)] comes first and occurs at $\ln \left(r_{2} / r_{1}\right)=\pi \sqrt{K_{3} / K_{1}}$.

For a nematic liquid crystal with negative dielectric anisotropy $\left(\epsilon_{a}<0\right)$ the flexoelectric effect reduces the critical voltage for the orientational transition. Using the MBBA material parameters at 

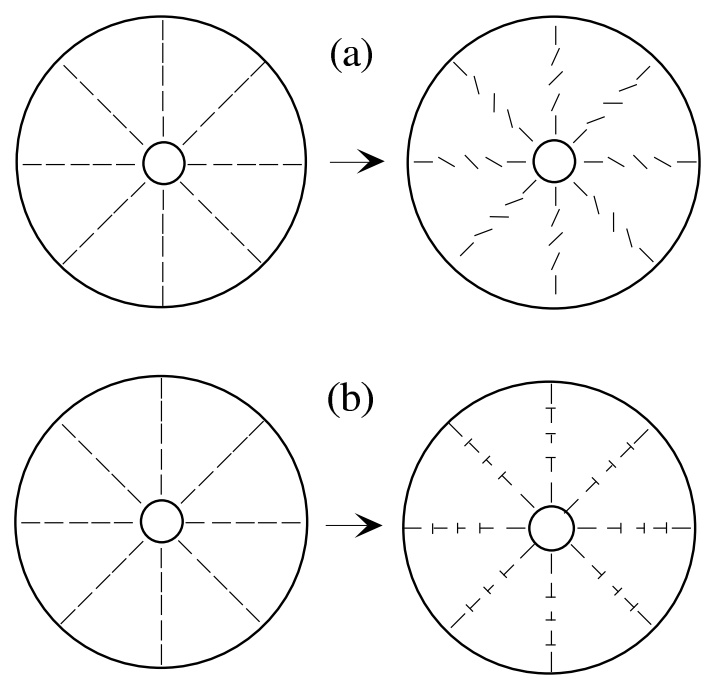

Figure 1: Orientational transition for $\delta n_{\phi}(\mathrm{a})$ and $\delta n_{z}(\mathrm{~b})$.
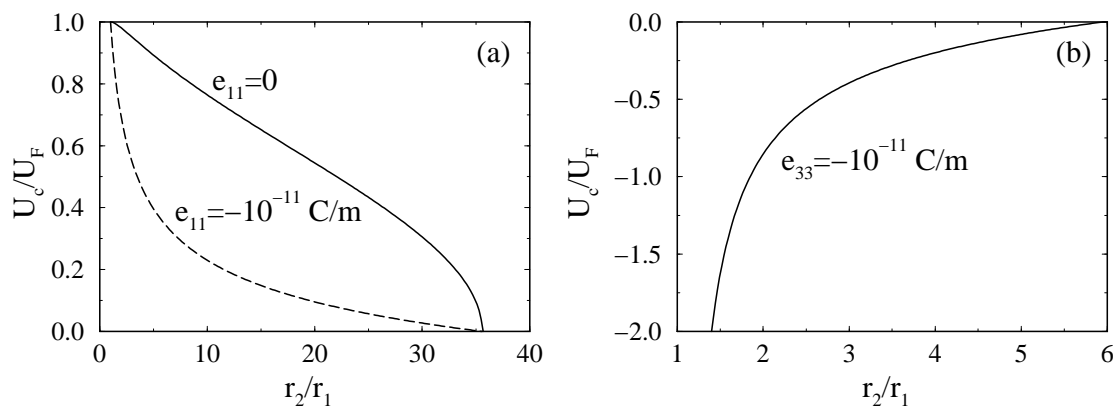

Figure 2: Critical voltage versus ratio of the radii for MBBA with homeotropic (a) and planar (b) anchoring.

$25^{\circ} \mathrm{C} K_{1}=6.66 \cdot 10^{-12} \mathrm{~N}, K_{2}=4.2 \cdot 10^{-12} \mathrm{~N}, K_{3}=8.61 \cdot 10^{-12} \mathrm{~N}$, $\epsilon_{a}=-0.53$ and taking for the flexocoefficients the order of magnitude $e_{11}+e_{33}=e_{11}=-10^{-11} \mathrm{C} / \mathrm{m}$, 9, 10 we found that the transition corresponding to $\delta n_{z}$ perturbation has lower threshold [Fig. 2(a)]. In this case above $r_{2} / r_{1} \approx 35.6$ the orientational transition occurs without electric field.

II) Planar anchoring with boundary conditions

$$
\hat{\mathbf{n}}\left(r=r_{1}\right)=\hat{\mathbf{n}}\left(r=r_{2}\right)=(0,1,0) .
$$

Similar to the case of homeotropic orientation, small deviations from 


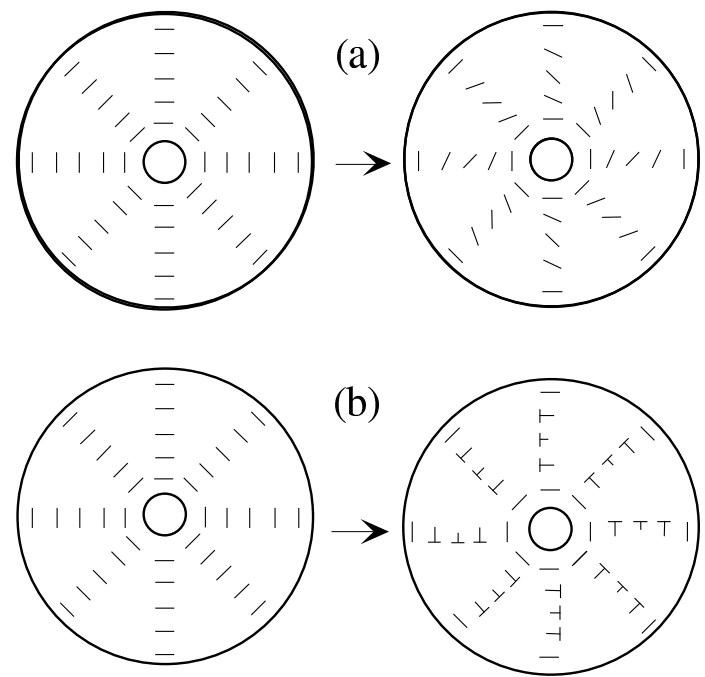

Figure 3: Orientational transition for $\delta n_{r}$ (a) and $\delta n_{z}(\mathrm{~b})$.

the exact solution $\hat{\mathbf{n}}_{0}=(0,1,0)$ of $(5)$ can be written as

$$
\hat{\mathbf{n}}=\hat{\mathbf{n}}_{0}+\delta \hat{\mathbf{n}}=\left[\delta n_{r} ; 1-\frac{1}{2}\left(\delta n_{r}^{2}+\delta n_{z}^{2}\right) ; \delta n_{z}\right]
$$

up to second order in the perturbations $\delta n_{r}, \delta n_{z}$ which depend only on $r$. Then the change of total free energy up to the second order in $\delta \hat{\mathbf{n}}$ is

$$
\begin{aligned}
\Delta \mathcal{F}_{\text {plan }} \equiv & \mathcal{F}\left(\hat{\mathbf{n}}_{0}\right)-\mathcal{F}\left(\hat{\mathbf{n}}_{0}+\delta \hat{\mathbf{n}}\right)= \\
=2 \pi \int_{r_{1}}^{r_{2}}\{ & \frac{1}{2} K_{1}\left[\delta n_{r}^{\prime 2}+\frac{1}{r^{2}} \delta n_{r}^{2}\right]+\frac{1}{2} K_{2}\left[\delta n_{z}^{\prime 2}+\frac{1}{r^{2}} \delta n_{z}^{2}\right]- \\
& \frac{1}{2} K_{3} \frac{1}{r^{2}}\left(\delta n_{r}^{2}+\delta n_{z}^{2}\right)-\frac{1}{2} \epsilon_{0} \epsilon_{a} \tilde{U}^{2} \frac{1}{r^{2}} \delta n_{r}^{2}- \\
& \left.\tilde{U} e_{11} \frac{1}{r^{2}} \delta n_{r}^{2}-\tilde{U} e_{33} \frac{1}{r^{2}}\left(\delta n_{r}^{2}+\delta n_{z}^{2}\right)\right\} r d r
\end{aligned}
$$

where the boundary conditions $\delta \hat{\mathbf{n}}\left(r=r_{1}\right)=\delta \hat{\mathbf{n}}\left(r=r_{2}\right)=0$ were taken into account.

The instability conditions can be obtained from the Euler-Lagrange equations

$$
\begin{aligned}
& K_{1} \frac{d^{2}}{d \alpha^{2}} \delta n_{r}+\left[K_{3}-K_{1}+\epsilon_{0} \epsilon_{a} \tilde{U}^{2}+2 \tilde{U}\left(e_{11}+e_{33}\right)\right] \delta n_{r}=0 \\
& K_{2} \frac{d^{2}}{d \alpha^{2}} \delta n_{z}+\left[2 K_{3}-K_{2}+2 \tilde{U} e_{33}\right] \delta n_{z}=0 .
\end{aligned}
$$


Here again the equations $(\sqrt{16})$, (17) are uncoupled and one has two independent conditions for the instability of initial planar orientation with respect to $\delta n_{r}$ and $\delta n_{z}$ perturbations (Fig. 3). The critical voltage $U_{c}$ for the orientational transition can be found from the solution of (16), (17)

$$
\begin{gathered}
\delta n_{r}: \quad \operatorname{sign}\left(\epsilon_{a}\right)\left(\frac{U_{c}}{U_{F_{1}}}\right)^{2}+\left(\frac{U_{c}}{U_{F_{1}}}\right) \frac{2\left(e_{11}+e_{33}\right) \ln \left(r_{2} / r_{1}\right)}{\pi \sqrt{K_{1} \epsilon_{0}\left|\epsilon_{a}\right|}}= \\
=1-\frac{K_{3}-K_{1}}{K_{1}}\left(\frac{\ln \left(r_{2} / r_{1}\right)}{\pi}\right)^{2}, \\
\delta n_{z}: \quad\left(\frac{U_{c}}{U_{F_{2}}}\right) \frac{2 e_{33} \ln \left(r_{2} / r_{1}\right)}{\pi \sqrt{K_{2} \epsilon_{0}\left|\epsilon_{a}\right|}}= \\
=1-\frac{2 K_{3}-K_{2}}{K_{2}}\left(\frac{\ln \left(r_{2} / r_{1}\right)}{\pi}\right)^{2},
\end{gathered}
$$

where $U_{F_{1}}=\pi \sqrt{K_{1} / \epsilon_{0}\left|\epsilon_{a}\right|}, U_{F_{2}}=\pi \sqrt{K_{2} / \epsilon_{0}\left|\epsilon_{a}\right|}$ are the threshold voltages for the splay and twist Fréedericksz transition in the case of a plane NLC layer, respectively. Without electric field one has the orientational transitions at the critical radius ratio $\ln \left(r_{2} / r_{1}\right)=$ $\pi \sqrt{K_{1} /\left(K_{3}-K_{1}\right)}$ [Fig. 3) (a)] and $\ln \left(r_{2} / r_{1}\right)=\pi \sqrt{K_{2} /\left(2 K_{3}-K_{2}\right)}$ [Fig. 3(b)].

For NLC with $\epsilon_{a}>0$ the flexoelectric effect reduces the critical voltage for the transition corresponding to $\delta n_{r}$ perturbations similar to that found in the case of homeotropically oriented nematics with $\epsilon_{a}<0$ [Fig. 2(a)]. Equation (19) demonstrates a new feature of the cylindrical geometry, namely, the possibility of an orientational transition caused purely by the flexoelectric effect. In Figure 2(b) the threshold voltage corresponding to the flexoelectric instability ( $\delta n_{z}$ perturbation) as a function of the ratio of the radii is plotted for the MBBA material parameters and $e_{33}=-10^{-11} \mathrm{C} / \mathrm{m}$. Above $r_{2} / r_{1} \approx 5.96$ the transition takes place without electric field.

Thus, the analysis of the orientational transitions in a NLC confined between two coaxial cylinders shows the strong influence of the flexoelectric effect on the critical voltage. For planar boundary conditions a pure flexoelectric instability is found. In this case the polarity of critical voltage depends only on the sign of the flexo-coefficient $e_{33}$ 
which allows to determine the value and the sign of $e_{33}$ in a simple experiment. Choosing the radius ratio of the cylinders close to the critical (above which the transition occurs without electric field) one can make the threshold voltage for the flexoelectric-induced orientational transition very small and reduce the possible influence of the electric current in a not sufficiently clean NLC materials.

\section{Acknowledgments}

We thank L. Kramer for fruitful discussions and a critical reading of the manuscript. A.K. wishes to acknowledge the hospitality of the University of Bayreuth. Financial support from DFG Grant Kr-690/14-1 and INTAS Grant 96-498 is gratefully acknowledged. M.Kh. is also grateful to the INTAS for fellowship grant YSF 994035 .

\section{References}

[1] P.E. Cladis and M. Kleman, J. Phys. (Paris), 33, 591 (1972).

[2] C.E. Williams, P.E. Cladis and M. Kleman, Mol. Cryst. Liq. Cryst., 21, 355 (1973).

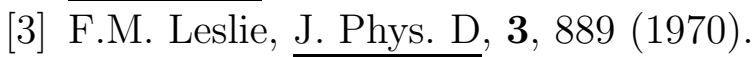

[4] U.D. Kini, Pramana, 7, 223 (1977).

[5] H. Tsuru, J. Phys. Soc. Jap., 59, 1600 (1990).

[6] N. Aoki, Y. Ohki and K. Yahagi, Jap. J. Appl. Phys., 18, 523 (1979).

[7] D.R.M. Williams and A. Halperin, Phys. Rev. E, 48, R2366 (1993).

[8] R.B. Meyer, Phys. Rev. Lett., 22, 918 (1969).

[9] I. Dozov, Ph. Martinot-Lagarde and G. Durand, J. Phys. Lett. (France), 43, L365 (1982).

[10] N.V. Madhusudana and G. Durand, J. Phys. Lett. (France), 46, L195 (1985). 